\title{
External arguments for the chaotic bands calculation in the Mandelbrot set
}

\author{
G. Pastor*, M. Romera G. Álvarez and F. Montoya, \\ Instituto de Física Aplicada, Consejo Superior de Investigaciones Científicas, \\ Serrano 144-28006 Madrid, Spain
}

\begin{abstract}
In this paper we study the chaotic bands of any Mandelbrot set hyperbolic component. We use external arguments in order to identify the hyperbolic components. If we use harmonics as a tool, we can calculate the chaotic bands. Indeed, as we clearly show here, the harmonics of the external arguments of a given hyperbolic component (gene) are the external arguments of the last appearance hyperbolic components of the chaotic bands corresponding to the gene. Likewise, we show that only if the hyperbolic component is a cardioid the infinite number of the chaotic bands of such a cardioid fills up all its chaotic region.
\end{abstract}

Key words: Mandelbrot set, external arguments, chaotic bands, hyperbolic components.

PACS: 05.45.+b, 47.20.Ky.

\section{Introduction}

We have widely studied the chaotic bands in the one-dimensional (1D) quadratic maps [1]. By using the harmonics, which were named Fourierharmonics in [1], we can calculate the structural components, which are the last appearance hyperbolic components of any chaotic band [1]. In [1] we have accomplished these calculations by using symbolic sequences [2,3] as identifiers [4] of the hyperbolic components [5] and Misiurewicz points [6-10]. However, in [11] we also have accomplished these calculations by using as identifiers the external arguments $[7,12-14]$ instead of symbolic sequences. In fact we use symbolic sequences in 1D quadratic maps and external arguments in the part of the Mandelbrot set antenna that intersects with the real axis. But 1D

* Corresponding author: Email: gerardo@iec.csic.es 
quadratic maps and the part of the Mandelbrot set antenna that intersects with the real axis are equivalent [1], hence we can use in both cases the two identifiers.

Likewise, we also have studied the chaotic bands in the Mandelbrot set $[15,16]$. In [15] we have used the shrubs in order to study the chaotic region of the Mandelbrot set, and in [16] we focus on the study of chaotic bands but with no calculation of their hyperbolic components.

In this paper we have used harmonics in order to calculate for the first time the last appearance hyperbolic components of the chaotic bands of any Mandelbrot set hyperbolic component. In some manner this paper finishes papers [11] and [16]. Indeed, in [15] harmonics are used in order to calculate the last appearance hyperbolic components of the chaotic bands of the real axis, but here we enlarge it to all the Mandelbrot set. In [16] we analyze the chaotic bands in all the Mandelbrot set with no calculation, but here we calculate the last appearance hyperbolic components. Therefore, the use of harmonics as a tool for the calculation of the last appearance hyperbolic components of the chaotic bands in all the Mandelbrot set can be considered the main contribution of this paper. More specifically, we clearly show that given the external arguments $\left(. \bar{a}_{1}, . \bar{a}_{2}\right)$ of a hyperbolic component of the Mandelbrot set, their successive harmonics, $H^{(i)}\left(\bar{a}_{1}, . \bar{a}_{2}\right), 2 \leq i \leq \infty$, calculate the external arguments of the last appearance hyperbolic components of the corresponding chaotic bands.

Let us finish by noting a couple of things. First, by using the exact words of J. Hubbard, our paper is based on "the use of computers as an experimental mathematical tool" [17]. That is to say, the methodology used is not the typical of the mathematicians, whose statements have to be proved, but that of experimental scientists. Indeed, we use the computer as an experimental tool and consequently the obtained results are valid while other experimental results do not contradict them. And second, we want to note that external rays are drawn by using an algorithm from W. Jung [18].

\section{Hyperbolic components on the real axis}

When external arguments are used as identifiers, let us remember how to calculate the harmonics of the antenna hyperbolic components that intersect with the real axis [11]. External arguments can be given in two forms: rational number or binary expansion. The formulas used to calculate harmonics are much simpler when using binary expansions rather than rational numbers. Indeed, as can be seen in [11], given a hyperbolic component, whose pair of external arguments expressed as binary expansions is $\left(. \bar{a}_{1}, . \bar{a}_{2}\right)$, the binary 
expansions of the pairs of the external arguments of the order $i$ harmonics of $\left(. \bar{a}_{1}, . \bar{a}_{2}\right)$ are given by a very simple formula:

$$
H^{(i)}\left(. \bar{a}_{1}, . \bar{a}_{2}\right)=(. \overline{a_{1}} \underbrace{a_{2} a_{2} \cdots a_{2}}_{i}, . \overline{a_{2}} \underbrace{a_{1} a_{1} \cdots a_{1}}_{i})
$$

One can also calculate the external arguments of the order $i$ harmonics in the normalized rational form [11]:

$$
H^{(i)}\left(\frac{a_{1}}{2^{p}-1}, \frac{a_{2}}{2^{p}-1}\right)=\left(\frac{a_{1} 2^{i p}+a_{2} \sum_{j=0}^{j=i-1} 2^{i p}}{2^{(i+1) p}-1}, \frac{a_{2} 2^{i p}+a_{1} \sum_{j=0}^{j=i-1} 2^{i p}}{2^{(i+1) p}-1}\right),
$$

but the formula is more complex, and therefore we use it much less.

Let us apply it to the main cardioid, whose pair of external arguments in binary expansion form is $(. \overline{0}, . \overline{1})$. We name the main cardioid $\mathbf{G}_{0}$, "G" since we consider it to be the gene of the harmonics, and " 0 " since the period is 1 (or $2^{0}$ ). The first harmonic is $(. \overline{01}, . \overline{10})$, which is the pair of external arguments of the first disc of the period doubling cascade, and the next harmonics are $(\overline{011}, . \overline{100}),(\overline{0111}, . \overline{1000}),(\overline{01111}, . \overline{10000}), \ldots$, which are the external arguments of the last appearance hyperbolic components (of periods $3,4,5$, $\ldots$ ) of the period $-2^{0}$ chaotic band $\mathbf{B}_{0}$. If we consider now the first disc of the period doubling cascade (of period $2^{1}$ ) as a new gene, $\mathbf{G}_{1}=(. \overline{01}, . \overline{10}$ ), its first harmonic is $(\overline{0110}, . \overline{1001})$, which is the pair of external arguments of the second disc of the period doubling cascade, and the next harmonics are $(\overline{.011010}, . \overline{100101}),(. \overline{01101010}, . \overline{10010101}),(. \overline{0110101010}, . \overline{1001010101}), \ldots$, which are the external arguments of the last appearance hyperbolic components (of periods $2 \cdot 3,2 \cdot 4,2 \cdot 5$ ) of the period- $2^{1}$ chaotic band $\mathbf{B}_{1}$. And so on; therefore, if we consider now the $n$-th disc of the period doubling cascade, whose period is $2^{n}$, as a new gene, $\mathbf{G}_{n}$, its first harmonic is the $(n+1)$-th disc of the period doubling cascade, and the next harmonics are the last appearance hyperbolic components of the period $-2^{n}$ chaotic band $\mathbf{B}_{n}$.

Of course, $0 \leq n \leq \infty$; therefore, $\mathbf{B}_{0}, \mathbf{B}_{1}, \mathbf{B}_{2}, \ldots, \mathbf{B}_{\infty}$ take up all the chaotic region, from the antenna tip at $c=-2$, that is the Misiurewicz point $m_{0}=$ $\operatorname{tip}(. \overline{0}, . \overline{1})=H^{(\infty)}(. \overline{0}, . \overline{1})=.0 \overline{1}=.1 \overline{0}$, to the Myrberg-Feigenbaum point $M F(. \overline{0}, . \overline{1})$, that separates the chaotic region and the periodic region.

In Fig. 1 we show a sketch of the period- $2^{0}$ main cardioid, $\mathbf{G}_{0}$, and the period doubling cascade discs $\mathbf{G}_{1}, \mathbf{G}_{2}, \mathbf{G}_{3}, \ldots$, whose periods are $2^{1}, 2^{2}, 2^{3}, \ldots$. We make evident that the chaotic bands $\mathbf{B}_{0}, \mathbf{B}_{1}, \mathbf{B}_{2}, \mathbf{B}_{3}, \ldots$ have their origins in the genes $\mathbf{G}_{0}, \mathbf{G}_{1}, \mathbf{G}_{2}, \mathbf{G}_{3}, \ldots$, because $H^{(i)}\left(\mathbf{G}_{0}\right), H^{(i)}\left(\mathbf{G}_{1}\right), H^{(i)}\left(\mathbf{G}_{2}\right)$, $H^{(i)}\left(\mathbf{G}_{3}\right), \ldots$, where $2 \leq i \leq \infty$, calculate the external arguments of the last appearance hyperbolic components of the chaotic bands $\mathbf{B}_{0}, \mathbf{B}_{1}, \mathbf{B}_{2}, \mathbf{B}_{3}, \ldots$ 


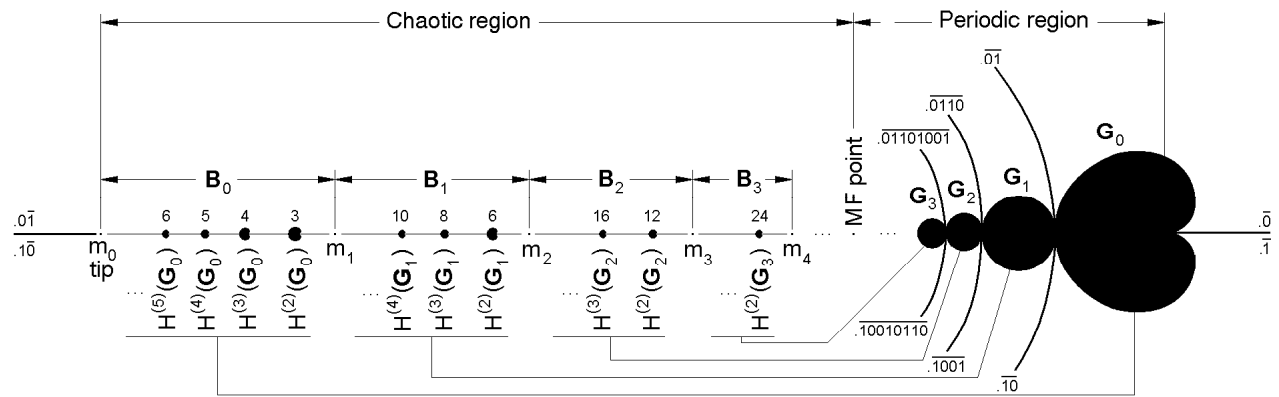

Fig. 1. Sketch of the period- $2^{0}$ main cardioid $\mathbf{G}_{0}$; of the period doubling cascade $\mathbf{G}_{1}$, $\mathbf{G}_{2}, \mathbf{G}_{3}, \ldots$, of periods $2^{1}, 2^{2}, 2^{3}, \ldots$; and the chaotic bands $\mathbf{B}_{0}, \mathbf{B}_{1}, \mathbf{B}_{2}, \mathbf{B}_{3}, \ldots$ These chaotic bands have their origins in the genes $\mathbf{G}_{0}, \mathbf{G}_{1}, \mathbf{G}_{2}, \mathbf{G}_{3}, \ldots$ since the harmonics $H^{(i)}\left(\mathbf{G}_{0}\right), H^{(i)}\left(\mathbf{G}_{1}\right), H^{(i)}\left(\mathbf{G}_{2}\right), H^{(i)}\left(\mathbf{G}_{3}\right), \ldots$, where $2 \leq i \leq \infty$, calculate the last appearance hyperbolic components of the chaotic bands $\mathbf{B}_{0}, \mathbf{B}_{1}, \mathbf{B}_{2}, \mathbf{B}_{3}, \ldots$

If instead of the main cardioid, we consider any other cardioid placed on the real axis, for example the only period-3 cardioid on the antenna, whose external arguments are $(\overline{011}, . \overline{100})$, we can calculate again the last appearance hyperbolic components of the chaotic bands of such a cardioid. Indeed, now $\mathbf{G}_{0}=(. \overline{011}, . \overline{100})$; and, if its harmonics are calculated according to Eq. (1), one obtains $H^{(1)}\left(\mathbf{G}_{0}\right)=(\overline{011100}, . \overline{100011})$, which is the first disc of the period doubling cascade, and $H^{(2)}\left(\mathbf{G}_{0}\right)=\left(. \overline{.011 \overline{100}^{2}}, . ._{100 \overline{011}^{2}}\right)$, $H^{(3)}\left(\mathbf{G}_{0}\right)=\left(\overline{.011 \overline{100}^{3}}, \quad . \overline{100 \overline{011}^{3}}\right), H^{(4)}\left(\mathbf{G}_{0}\right)=\left(. \overline{011 \overline{100}^{4}}, \quad .{\overline{100 \overline{011}^{4}}}^{4}, \ldots\right.$, which are the last appearance cardioids, whose periods are $3 \cdot 3,3 \cdot 4,3 \cdot 5$, $\ldots$, of the period- $3 \cdot 2^{0}$ chaotic band $\mathbf{B}_{0}$. Likewise, if one takes again the first disc of the period doubling cascade as $\mathbf{G}_{1}=(\overline{011100}, . \overline{100011})$, and if its harmonics are calculated according to Eq. (1), one obtains $H^{(1)}\left(\mathbf{G}_{1}\right)=(\overline{011100100011}, . \overline{100011011100})$, which is the second disc of the period doubling cascade, and $H^{(2)}\left(\mathbf{G}_{1}\right)=\left(.{\overline{011100 \overline{100011}^{2}}}^{2} .{\overline{100011 \overline{011100}^{2}}}^{2}\right.$, $H^{(3)}\left(\mathbf{G}_{1}\right)=\quad\left(\overline{.011100 \overline{100011}^{3}}, \quad . \overline{100011 \overline{011100}^{3}}\right), \quad H^{(4)}\left(\mathbf{G}_{1}\right)=$

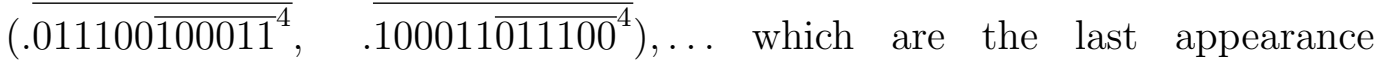
cardioids, whose periods are $2 \cdot 3 \cdot 3,2 \cdot 3 \cdot 4,2 \cdot 3 \cdot 5$, of the period- $3 \cdot 2^{1}$ chaotic band $\mathbf{B}_{1}$. And so on all the other chaotic bands can be calculated. In Fig. 2 we show a sketch of the chaotic bands of this period-3 hyperbolic component. The infinite number of chaotic bands $\mathbf{B}_{0}, \mathbf{B}_{1}, \mathbf{B}_{2}, \ldots$ take up all the chaotic region corresponding to the cardioid $(. \overline{011}, . \overline{100})$, from its tip, which is the Misiurewicz point $\operatorname{tip}(\overline{011}, . \overline{100})=H^{(\infty)}(\overline{.011}, . \overline{100})=(.011 \overline{100}, .100 \overline{011})$, to the Myrberg-Feigenbaum point $M F(. \overline{011}, . \overline{100})$.

As has just been seen in the two former cases that were placed on the real axis, by calculating the harmonics of a gene one obtains the external arguments of the last appearance hyperbolic components -that we name sometimes structural components- of the successive chaotic bands of the gene.

So far we only have dealt with hyperbolic components placed on the real axis 


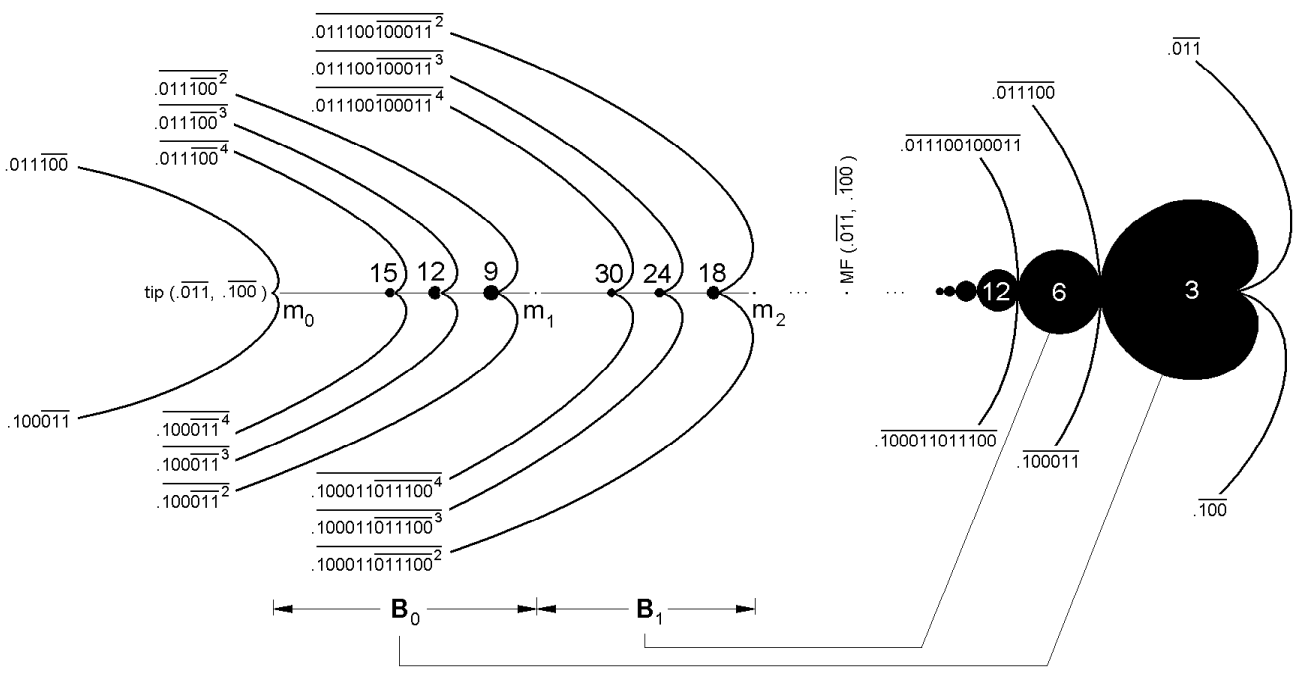

Fig. 2. Sketch of the only period-3 cardioid of the antenna, of its period doubling cascade and of its chaotic bands. The chaotic bands $\mathbf{B}_{0}, \mathbf{B}_{1}, \mathbf{B}_{2}, \ldots$ have their origins in the genes $\mathbf{G}_{0}, \mathbf{G}_{1}, \mathbf{G}_{2}, \ldots$ since the harmonics $H^{(i)}\left(\mathbf{G}_{0}\right), H^{(i)}\left(\mathbf{G}_{1}\right), H^{(i)}\left(\mathbf{G}_{2}\right), \ldots$, where $2 \leq i \leq \infty$, calculate the last appearance hyperbolic components of the chaotic bands $\mathbf{B}_{0}, \mathbf{B}_{1}, \mathbf{B}_{2}, \ldots$

of the Mandelbrot set. However we shall use now formulas (1) and (2) in order to calculate the harmonics of hyperbolic components placed out of the real axis; i. e., placed in any region of the Mandelbrot set.

As can be seen experimentally, the external arguments calculated are the last appearance hyperbolic components of the chaotic bands of the gene. Indeed, let $\left(. \bar{a}_{1}, . \bar{a}_{2}\right)$ be the external arguments of a given gene. By using the computer program of W. Jung [18], one can see the hyperbolic components corresponding to the successive harmonics of the external arguments of the gene: $H^{(i)}\left(. \bar{a}_{1}, . \bar{a}_{2}\right), 2 \leq i \leq \infty$. As we shall see, these harmonics are the last appearance hyperbolic components of the chaotic band corresponding to the gene.

\section{Hyperbolic components out of the real axis}

Let us see the period-3 primary hyperbolic component $\frac{1}{3}$ (it is primary because it is directly attached to the main cardioid [15]). Its external arguments are $\mathbf{G}_{0}=(. \overline{001}, \overline{010})$. If the harmonics are calculated, one has $H^{(1)}\left(\mathbf{G}_{0}\right)=(\overline{001010}, \overline{010001})$, which is the first disc of the period doubling cascade, and $H^{(2)}\left(\mathbf{G}_{0}\right)=\left(.{\overline{.001 \overline{010}^{2}}}^{2} .{\overline{.010 \overline{001}^{2}}}^{2}\right.$, $H^{(3)}\left(\mathbf{G}_{0}\right)=\left(\overline{.001 \overline{010}^{3}}, \overline{.010 \overline{001}^{3}}\right), H^{(4)}\left(\mathbf{G}_{0}\right)=\left(\overline{.001 \overline{010}^{4}}, \overline{.010 \overline{001}^{4}}\right), \ldots$, which are the last appearance cardioids (whose periods are $33,34,35$, ) of the period- $3 \cdot 2^{0}$ chaotic band $\mathbf{B}_{0}\left(\frac{1}{3}\right)$. Likewise, by considering again the 
first disc of the period doubling cascade as a gene $\mathbf{G}_{1}=(. \overline{001010}, . \overline{010001})$, if its harmonics are calculated according to Eq. (1) one obtains $H^{(1)}\left(\mathbf{G}_{1}\right)=(\overline{001010010001}, \overline{010001001010})$, which is the second disc of the period doubling cascade, and $H^{(2)}\left(\mathbf{G}_{1}\right)=\left(. \overline{001010010001}^{2}, .{\overline{010001 \overline{001010}^{2}}}^{2}\right.$, $H^{(3)}\left(\mathbf{G}_{1}\right)=\quad\left(\overline{.001010 \overline{010001}^{3}}, \quad .010001 \overline{001010}^{3}\right), \quad H^{(4)}\left(\mathbf{G}_{1}\right)=$ $\left(\overline{.001010 \overline{010001}^{4}}, \quad .{\overline{010001 \overline{001010}^{4}}}^{4}, \ldots\right.$, which are the last appearance cardioids of the period- $3 \cdot 2^{1}$ chaotic band $\mathbf{B}_{1}\left(\frac{1}{3}\right)$. And so on the successive chaotic bands can be calculated.

In Fig. 3 one can see a sketch of the hyperbolic components calculated that can be located by using the before cited tools [18]. The $\operatorname{shrub}\left(\frac{1}{3}\right)$ [15], which is the shrub of the primary hyperbolic component $\frac{1}{3}$ that we are dealing with, is characterized by a decoration with 3 branches around each node. As explained in [15], if a number is associated with each branch, one can calculate the period of the hyperbolic component representative (which are the last appearance hyperbolic components) of any branch of the shrub. The branch 0 , also called main branch, or $\operatorname{shrub}\left(\frac{1}{3}\right)$, has been studied in [16]. As can be seen in Fig. 3, the chaotic bands are placed just in this main branch of the shrub, occupying it completely. The limits are the main node, which is the Misiurewicz point $m_{0}=H^{(\infty)}(. \overline{001}, . \overline{010})=(.001 \overline{010}, .010 \overline{001})$, and the Myrberg-Feigenbaum point $M F\left(\frac{1}{3}\right)$.

But in the same way that one has dealt with a primary hyperbolic component, one could have dealt with a secondary hyperbolic component [15]. For example, let us see the period-12 secondary hyperbolic component $\frac{1}{3} \cdot \frac{1}{4}$ (it is secondary because it is directly attached to a primary hyperbolic component, the $\frac{1}{3}$ [15]). Its external arguments are: $\mathbf{G}_{0}=(\overline{001001001010}, \overline{001001010001})$. If the harmonics are calculated, $H^{(1)}\left(\mathbf{G}_{0}\right)=(. \overline{001001001010001001010001}, \quad . \overline{001001010001001001001010})$ is the first disc of he period doubling cascade, and $H^{(2)}\left(\mathbf{G}_{0}\right)=$

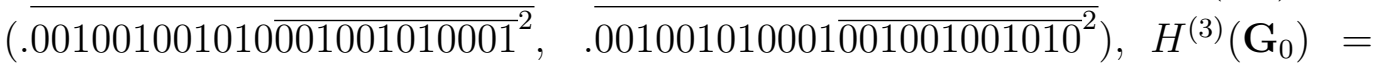
$\left(. \overline{.001001001010 \overline{001001010001}}{ }^{3}, \quad .001001010001 \overline{001001001010^{3}}\right), \quad H^{(4)}\left(\mathbf{G}_{0}\right)=$

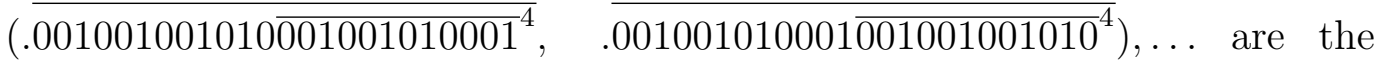
last appearance cardioids (whose periods are $12 \cdot 3,12 \cdot 4,12 \cdot 5, \ldots$ ) of the period-12 $\cdot 2^{0}$ chaotic band $\mathbf{B}_{0}\left(\frac{1}{3} \cdot \frac{1}{4}\right)$. Likewise, by considering again the first disc of the period doubling cascade as a gene $\mathbf{G}_{1}=$ $(\overline{001001001010001001010001}, \overline{001001010001001001001010})$, if the harmonics are calculated according to Eq. (1) one obtains $H^{(1)}\left(\mathbf{G}_{1}\right)$, which is the second disc of the period doubling cascade (the binary expansion is not given because is too large), and $H^{(2)}\left(\mathbf{G}_{1}\right), H^{(3)}\left(\mathbf{G}_{1}\right), H^{(4)}\left(\mathbf{G}_{1}\right), \ldots$ (the binary expansions are not given either because are even larger) which are the last appearance cardioids (whose periods are $12 \cdot 2 \cdot 3,12 \cdot 2 \cdot 4,12 \cdot 2 \cdot 5, \ldots$ ) of the period$12 \cdot 2^{1}$ chaotic band $\mathbf{B}_{1}\left(\frac{1}{3} \cdot \frac{1}{4}\right)$. And so on the successive chaotic bands can be calculated. 


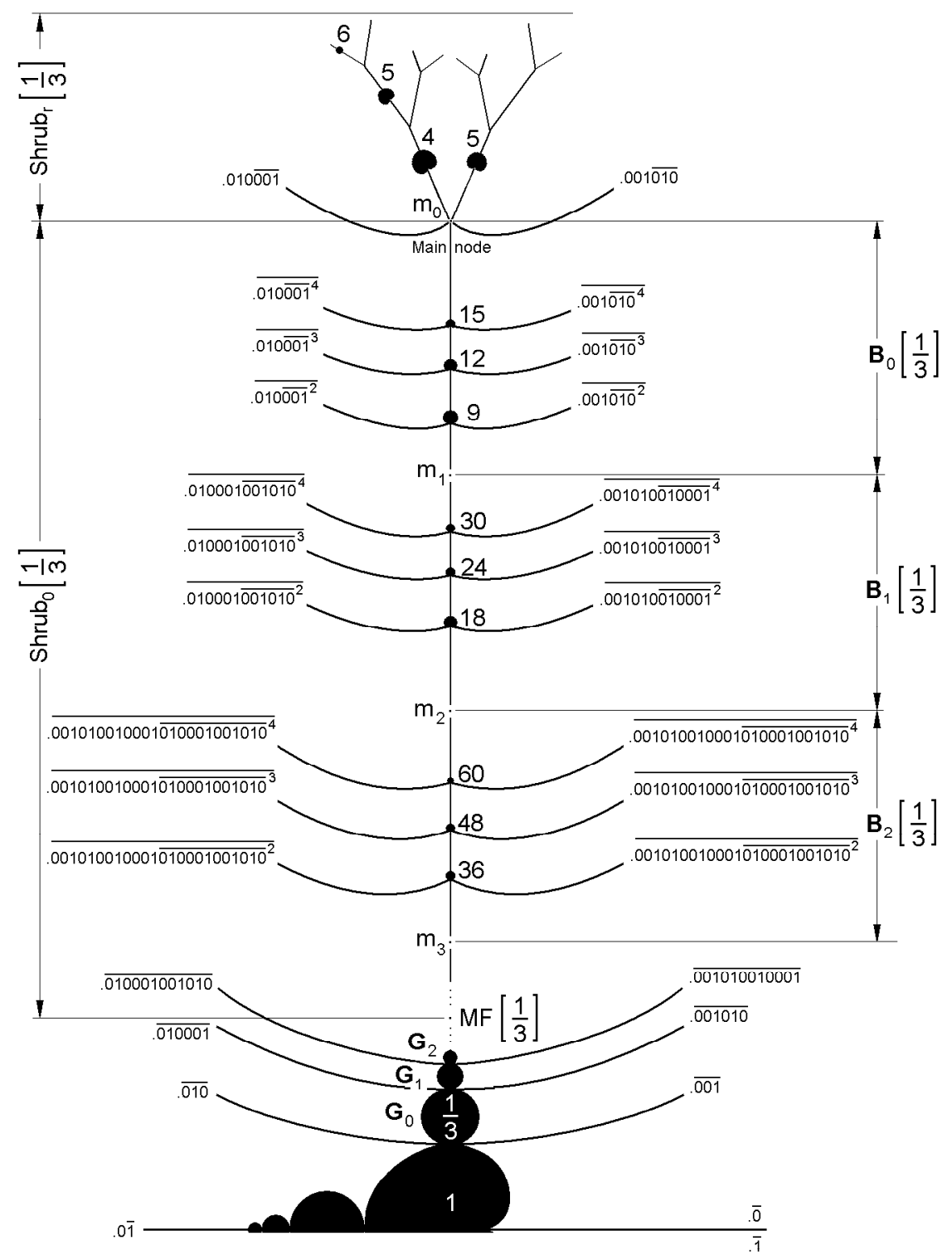

Fig. 3. Sketch of the primary hyperbolic component $\mathbf{G}_{0}=\frac{1}{3}$, of its period doubling cascade, and of $\operatorname{shrub}\left(\frac{1}{3}\right)$. Detail of $\operatorname{shrub}\left(\frac{1}{3}\right)$, completely occupied by the infinity of chaotic bands $\mathbf{B}_{0}\left(\frac{1}{3}\right), \mathbf{B}_{1}\left(\frac{1}{3}\right), \mathbf{B}_{2}\left(\frac{1}{3}\right), \ldots$ These chaotic bands have their origins in $\mathbf{G}_{0}\left(\frac{1}{3}\right), \mathbf{G}_{1}\left(\frac{1}{3}\right), \mathbf{G}_{2}\left(\frac{1}{3}\right), \ldots$ since the harmonics of the genes calculate the last appearance hyperbolic components of the corresponding chaotic bands.

Fig. 4 shows a sketch of the hyperbolic components calculated. The $\operatorname{shrub}\left(\frac{1}{3} \cdot \frac{1}{4}\right)$, which is the shrub of the secondary hyperbolic component we are dealing with, has two parts: the $\operatorname{shrub}_{0}\left(\frac{1}{3} \cdot \frac{1}{4}\right)$ and the $\operatorname{shrub}_{r}\left(\frac{1}{3} \cdot \frac{1}{4}\right)$. The $\operatorname{shrub}_{r}\left(\frac{1}{3} \cdot \frac{1}{4}\right)$ has two subshrubs, the first one characterized by a decoration that has 4 branches around each node, and the second one characterized by a decoration that has 3 branches around each node [15]. Again, as Fig. 4 shows, the chaotic bands $\mathbf{B}_{0}\left(\frac{1}{3} \cdot \frac{1}{4}\right), \mathbf{B}_{1}\left(\frac{1}{3} \cdot \frac{1}{4}\right), \mathbf{B}_{2}\left(\frac{1}{3} \cdot \frac{1}{4}\right), \ldots$ are placed on the $\operatorname{shrub}_{0}\left(\frac{1}{3} \cdot \frac{1}{4}\right)$, by occupying it completely. The limits of $\operatorname{shrub}\left(\frac{1}{3} \cdot \frac{1}{4}\right)$ are the main node, that is the Misiurewicz point $m_{0}=$ 


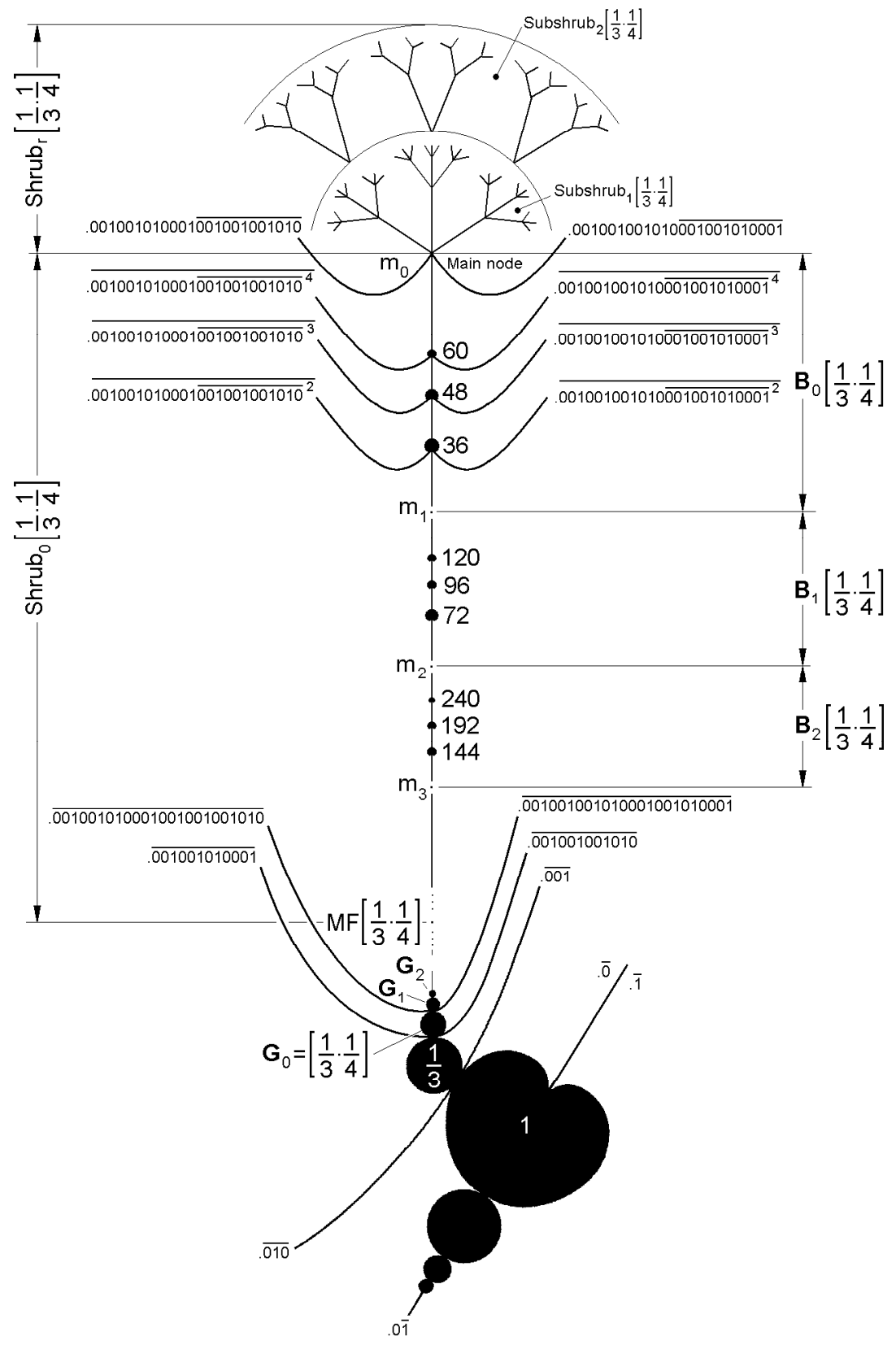

Fig. 4. Sketch of the primary hyperbolic component $\mathbf{G}_{0}=\frac{1}{3} \cdot \frac{1}{4}$, of its period doubling cascade, and of $\operatorname{shrub}\left(\frac{1}{3} \cdot \frac{1}{4}\right)$. The infinity of chaotic bands $\mathbf{B}_{0}\left(\frac{1}{3} \cdot \frac{1}{4}\right), \mathbf{B}_{1}\left(\frac{1}{3} \cdot \frac{1}{4}\right)$, $\mathbf{B}_{2}\left(\frac{1}{3} \cdot \frac{1}{4}\right), \ldots$ occupy completely the $\operatorname{shrub}\left(\frac{1}{3} \cdot \frac{1}{4}\right) . \mathbf{G}_{0}\left(\frac{1}{3} \cdot \frac{1}{4}\right)$ is the gene of $\mathbf{B}_{0}\left(\frac{1}{3} \cdot \frac{1}{4}\right)$, $\mathbf{G}_{1}\left(\frac{1}{3} \cdot \frac{1}{4}\right)$ is the gene of $\mathbf{B}_{1}\left(\frac{1}{3} \cdot \frac{1}{4}\right)$, and so on, since the harmonics of the first ones calculate the last appearance hyperbolic components of the last ones.

$H^{(\infty)}\left(\frac{1}{3} \cdot \frac{1}{4}\right)=(.001001001010 \overline{001001010001}, .001001010001 \overline{001001001010})$ and the Myrberg-Feigenbaum point $M F\left(\frac{1}{3} \cdot \frac{1}{4}\right)$.

We could consider now the cases of a tertiary, quaternary, hyperbolic component, but it is not worth the trouble because the exposition is very tedious and repetitive, and in addition, we obtain always the same. Thus, for the case 
of an N-ary hyperbolic component $\frac{q_{1}}{p_{1}} \cdots \frac{q_{N}}{p_{N}}$, the $\operatorname{shrub}\left(\frac{q_{1}}{p_{1}} \cdots \frac{q_{N}}{p_{N}}\right)$ splits as always in two parts [15]: The $\operatorname{shrub}_{r}\left(\frac{q_{1}}{p_{1}} \cdots \frac{q_{N}}{p_{N}}\right)$ (made up of N subshrubs) and the $\operatorname{shrub}_{0}\left(\frac{q_{1}}{p_{1}} \cdots \frac{q_{N}}{p_{N}}\right)$, which is the branch 0 or the main branch of the shrub. Again, the chaotic bands $\mathbf{B}_{0}\left(\frac{q_{1}}{p_{1}} \cdots \frac{q_{N}}{p_{N}}\right), \mathbf{B}_{1}\left(\frac{q_{1}}{p_{1}} \cdots \frac{q_{N}}{p_{N}}\right), \mathbf{B}_{2}\left(\frac{q_{1}}{p_{1}} \cdots \frac{q_{N}}{p_{N}}\right)$, occupy completely the $\operatorname{shrub}\left(\frac{q_{1}}{p_{1}} \cdots \frac{q_{N}}{p_{N}}\right)$, whose limits are the main node $m_{0}\left(\frac{q_{1}}{p_{1}} \cdots \frac{q_{N}}{p_{N}}\right)$ and $M F\left(\frac{q_{1}}{p_{1}} \cdots \frac{q_{N}}{p_{N}}\right)$.

There is a significant difference between the cases seen in section 2, whose hyperbolic components are on the real axis, and those seen in the section 3, that are not on the real axis. Indeed, in the real axis cases, the set of the infinite number of chaotic bands $\mathbf{B}_{0}, \mathbf{B}_{1}, \mathbf{B}_{2}, \ldots$ fills up all the chaotic region of the cardioid treated, which extends from its Myrberg-Feigenbaum point to the tip. On the contrary, in the cases out of the real axis, the set of the infinite number of chaotic bands $\mathbf{B}_{0}, \mathbf{B}_{1}, \mathbf{B}_{2}, \ldots$ fills up all the branch 0 of the shrub, or shrub $b_{0}$, which extends from the Myrberg-Feigenbaum point to the main node. However, the $s h r u b_{0}$ is not all the chaotic region since their is also other part, the shrub.

Hence, we can ask ourselves: is this difference due to being or not being on the real axis? The answer is no. The true reason is the following: on the real axis the set of the infinite number of chaotic bands $\mathbf{B}_{0}, \mathbf{B}_{1}, \mathbf{B}_{2}, \ldots$ fills up all the chaotic region because in such cases hyperbolic components are cardioids. Cardioids are born in a tangent bifurcation, which is the origin of the periodic region, that in turn extends through its period doubling cascade up to the MyrbergFeigenbaum point. Given that when we start from a cardioid we cover all the periodic region, the set of infinite number of chaotic bands $\mathbf{B}_{0}, \mathbf{B}_{1}, \mathbf{B}_{2}, \ldots$ also fills up the all the chaotic region. On the contrary, in the two cases seen out of the real axis, the hyperbolic components are not cardioids but discs, one of them is a primary hyperbolic component and the other one is a secondary hyperbolic component. Hence, when one covers its period doubling cascade up to the Myrberg-Feigenbaum point all the periodic region from the origin is not filled up. In the primary hyperbolic component case there is one hyperbolic component (the cardioid) to fill up the periodic region, and in the secondary hyperbolic component case there are two hyperbolic components (the primary hyperbolic component and the cardioid). Therefore, in the primary hyperbolic component case there is a shrub $r$ with only one subshrub, and in the secondary hyperbolic component case there is a $s h r u b_{r}$ with two subshrubs.

In Fig. 5 one can see an example that shows what we have just said. Fig. 5(a) shows the $\operatorname{shrub}\left(\frac{1}{3}\right)$ [15]. Fig. 5(b) shows the chaotic bands of the representative of the $\operatorname{shrub}\left(\frac{1}{3}\right)$ branch 1 , which is the period-4 cardioid that is in the rectangle shown in Fig. 5(a). The external arguments of the cardioid are: $\mathbf{G}_{0}=(\overline{0011}, \overline{0100})$. If the harmonics are calculated according to Eq. $(1), H^{(1)}\left(\mathbf{G}_{0}\right)=(\overline{.00110100}, \overline{01000011})$ is the first disc of the pe- 


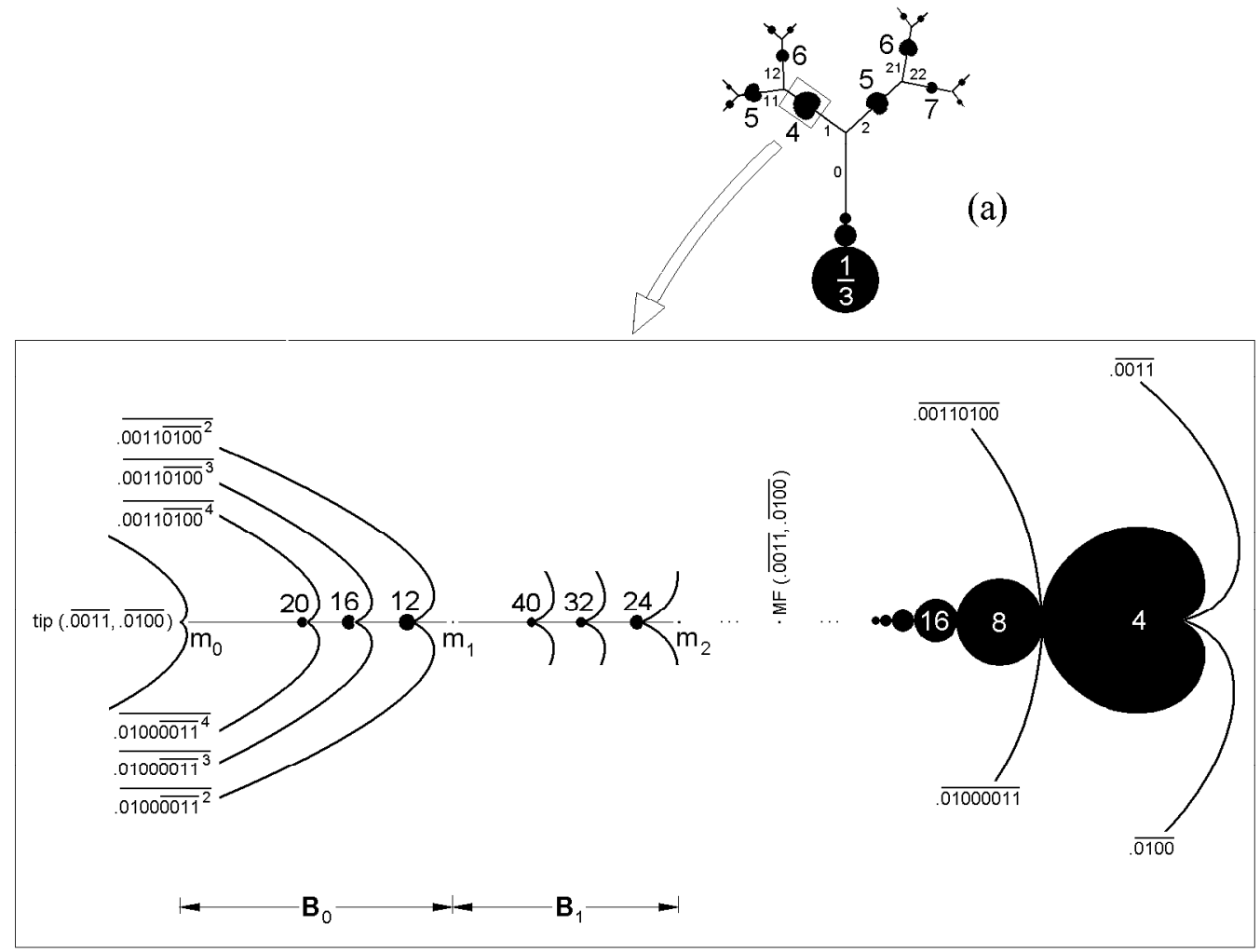

(b)

Fig. 5. (a) Sketch of $\operatorname{shrub}\left(\frac{1}{3}\right)$ showing the number of the branches and the periods of the hyperbolic components representatives. (b) Sketch of the branch 1 representative of $\operatorname{shrub}\left(\frac{1}{3}\right)$, of period 4 , which was inside a rectangle in (a). The infinity of the chaotic bands $\mathbf{B}_{0}, \mathbf{B}_{1}, \mathbf{B}_{2}, \ldots$ occupy all the chaotic region, from the tip to the MF point. $\mathbf{B}_{0}$ has its origin in the period- 4 cardioid $\mathbf{G}_{0}, \mathbf{B}_{1}$ in the period- 8 first disc of the period doubling cascade $\mathbf{G}_{1}$, and so on.

riod doubling cascade, and $H^{(2)}\left(\mathbf{G}_{0}\right)=\left(\overline{.0011 \overline{0100}^{2}}, \overline{.0100 \overline{0011}^{2}}\right), H^{(3)}\left(\mathbf{G}_{0}\right)=$

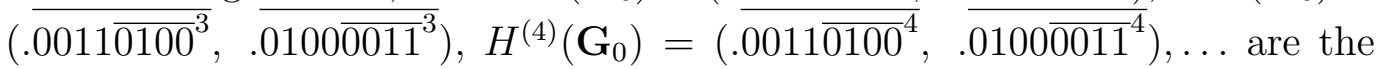
last appearance cardioids (with periods $4 \cdot 3,4 \cdot 4,4 \cdot 5, \ldots$ ) of the period- $4 \cdot 2^{0}$ chaotic band $\mathbf{B}_{0}$. One acts in the same way in order to calculate the other chaotic bands. Now the first disc of the period doubling cascade, with period 42 , is considered the gene $\mathbf{G}_{1}=(\overline{.00110100}, . \overline{01000011})$ of the period- $4 \cdot 2^{1}$ chaotic band $\mathbf{B}_{1}$; and the nth disc of the period doubling cascade, with period $4 \cdot 2^{n}$, is considered the gene of the period $-4 \cdot 2^{n}$ chaotic band $\mathbf{B}_{n}$. This representative of the branch 1 we are dealing with, whose period is 4 , is not on the real axis, and however the set of its infinite number of chaotic bands fills up all the chaotic region (there is no shrub remainder) because this representative is a cardioid. 


\section{Conclusions}

We studied the chaotic bands of any hyperbolic component of the Mandelbrot set.

We used the external arguments as the identifiers of the hyperbolic components. These external arguments have been given in the binary expansion form in order to make exposition easier.

We used the harmonics to calculate for the first time the external arguments of the last appearance hyperbolic components of the chaotic bands. Thus, the successive chaotic bands can be calculated from the gene of each band.

The starting hyperbolic component, the gene $\mathbf{G}_{0}$, is the generator of the chaotic band $\mathbf{B}_{0}$, and the successive discs of its period doubling cascade, $\mathbf{G}_{1}, \mathbf{G}_{2}, \mathbf{G}_{3}, \ldots$, are the generators of the chaotic bands $\mathbf{B}_{1}, \mathbf{B}_{2}, \mathbf{B}_{3}, \ldots$.

As we clearly show here, when we start from a cardioid, the infinite number of chaotic bands of a hyperbolic component hc, $\mathbf{B}_{0}, \mathbf{B}_{1}, \mathbf{B}_{2}, \mathbf{B}_{3}, \ldots$, fills up all the chaotic region of hc, the shrub(hc). This is not so when the starting hyperbolic component is a disc, in whose case the infinite number of chaotic bands fills up only the $s h r u b_{0}(h c)$.

\section{Acknowledgements}

This work was supported by Ministerio de Ciencia y Tecnología of Spain, research grants TIC2001-0586 and SEG2004-02418.

\section{References}

[1] G. Pastor, M. Romera and F. Montoya, Physical Review E 56 (1997) 1476.

[2] N. Metropolis, M. L. Stein and P. R. Stein, J. Combinat. Theory, 15 (1973) 25

[3] G. Pastor, M. Romera, J.C. Sanz-Martn and F. Montoya, Physica A 256 (1998) 369.

[4] G. Pastor, M. Romera, G. Álvarez and F. Montoya, Chaos, Solitons and Fractals 18 (2003) 899.

[5] B. Branner, Proc. Symposia Appl. Math. 39 (1989) 75-105.

[6] Misiurewicz M and Nitecki Z. Memoirs of the Am. Math. Soc. 94 (1991) n 456. 
[7] A. Douady and J.H. Hubbard, Étude Dynamique des Polynmes Complexes (Publ. Math. d'Orsay, Part I No. 84-02, 1984; Part II No. 85-04, 1985).

[8] M. Romera, G. Pastor and F. Montoya, Physica A 232: (1996) 517.

[9] G. Pastor, M. Romera and F. Montoya, Physica A 232 (1996) 536.

[10] G. Pastor, M. Romera, G. Álvarez and F. Montoya, Physica A 292 (2001) 207.

[11] G. Pastor, M. Romera, G. Álvarez and F. Montoya, Physica D 171 (2002) 52.

[12] A. Douady and J.H. Hubbard, C. R. Acad. Sc. Paris 294 (1982) 123.

[13] A. Douady, in Chaotic Dynamics and Fractals, edited by M. Barnsley and S. G. Demko, Academic Press, New York 1986, p.155.

[14] A. Douady. Invited contribution in H.-O. Peitgen and P.H. Richter, The Beauty of Fractals, Springer, Berlin 1986, p.161.

[15] M. Romera, G. Pastor, G. Álvarez and F. Montoya, Int. J. of Bifurcation and Chaos, 13(8) (2003) 2279.

[16] G. Pastor, M. Romera, G. Álvarez and F. Montoya, Computer and Graphics 28 (2004) 779.

[17] J. Hubbard, in The Mandelbrot set, Theme and Variations, edited by Tan Lei, Cambridge University Press, Cambridge 2000. Preface xiii.

[18] W. Jung, Programs for dynamical systems (1999), http://www.iram.rwthaachen.de/ jung/indexp.html 


\section{Figure captions}

Fig. 1: Sketch of the period- $2^{0}$ main cardioid $\mathbf{G}_{0}$; of the period doubling cascade $\mathbf{G}_{1}, \mathbf{G}_{2}, \mathbf{G}_{3}, \ldots$, of periods $2^{1}, 2^{2}, 2^{3}, \ldots$; and the chaotic bands $\mathbf{B}_{0}$, $\mathbf{B}_{1}, \mathbf{B}_{2}, \mathbf{B}_{3}, \ldots$ These chaotic bands have their origins in the genes $\mathbf{G}_{0}, \mathbf{G}_{1}$, $\mathbf{G}_{2}, \mathbf{G}_{3}, \ldots$ since the harmonics $H^{(i)}\left(\mathbf{G}_{0}\right), H^{(i)}\left(\mathbf{G}_{1}\right), H^{(i)}\left(\mathbf{G}_{2}\right), H^{(i)}\left(\mathbf{G}_{3}\right), \ldots$, where $2 \leq i \leq \infty$, calculate the last appearance hyperbolic components of the chaotic bands $\mathbf{B}_{0}, \mathbf{B}_{1}, \mathbf{B}_{2}, \mathbf{B}_{3}, \ldots$

Fig. 2: Sketch of the only period-3 cardioid of the antenna, of its period doubling cascade and of its chaotic bands. The chaotic bands $\mathbf{B}_{0}, \mathbf{B}_{1}, \mathbf{B}_{2}, \ldots$ have their origins in the genes $\mathbf{G}_{0}, \mathbf{G}_{1}, \mathbf{G}_{2}, \ldots$ since the harmonics $H^{(i)}\left(\mathbf{G}_{0}\right)$, $H^{(i)}\left(\mathbf{G}_{1}\right), H^{(i)}\left(\mathbf{G}_{2}\right), \ldots$, where $2 \leq i \leq \infty$, calculate the last appearance hyperbolic components of the chaotic bands $\mathbf{B}_{0}, \mathbf{B}_{1}, \mathbf{B}_{2}, \ldots$

Fig. 3: Sketch of the primary hyperbolic component $\mathbf{G}_{0}=\frac{1}{3}$, of its period doubling cascade, and of $\operatorname{shrub}\left(\frac{1}{3}\right)$. Detail of $\operatorname{shrub}\left(\frac{1}{3}\right)$, completely occupied by the infinity of chaotic bands $\mathbf{B}_{0}\left(\frac{1}{3}\right), \mathbf{B}_{1}\left(\frac{1}{3}\right), \mathbf{B}_{2}\left(\frac{1}{3}\right), \ldots$ These chaotic bands have their origins in $\mathbf{G}_{0}\left(\frac{1}{3}\right), \mathbf{G}_{1}\left(\frac{1}{3}\right), \mathbf{G}_{2}\left(\frac{1}{3}\right), \ldots$ since the harmonics of the genes calculate the last appearance hyperbolic components of the corresponding chaotic bands.

Fig. 4: Sketch of the primary hyperbolic component $\mathbf{G}_{0}=\frac{1}{3} \cdot \frac{1}{4}$, of its period doubling cascade, and of $\operatorname{shrub}\left(\frac{1}{3} \cdot \frac{1}{4}\right)$. The infinity of chaotic bands $\mathbf{B}_{0}\left(\frac{1}{3} \cdot \frac{1}{4}\right)$, $\mathbf{B}_{1}\left(\frac{1}{3} \cdot \frac{1}{4}\right), \mathbf{B}_{2}\left(\frac{1}{3} \cdot \frac{1}{4}\right), \ldots$ occupy completely the $\operatorname{shrub}_{0}\left(\frac{1}{3} \cdot \frac{1}{4}\right) \cdot \mathbf{G}_{0}\left(\frac{1}{3} \cdot \frac{1}{4}\right)$ is the gene of $\mathbf{B}_{0}\left(\frac{1}{3} \cdot \frac{1}{4}\right), \mathbf{G}_{1}\left(\frac{1}{3} \cdot \frac{1}{4}\right)$ is the gene of $\mathbf{B}_{1}\left(\frac{1}{3} \cdot \frac{1}{4}\right)$, and so on, since the harmonics of the first ones calculate the last appearance hyperbolic components of the last ones.

Fig. 5: (a) Sketch of $\operatorname{shrub}\left(\frac{1}{3}\right)$ showing the number of the branches and the periods of the hyperbolic components representatives. (b) Sketch of the branch 1 representative of $\operatorname{shrub}\left(\frac{1}{3}\right)$, of period 4 , which was inside a rectangle in (a). The infinity of the chaotic bands $\mathbf{B}_{0}, \mathbf{B}_{1}, \mathbf{B}_{2}, \ldots$ occupy all the chaotic region, from the tip to the MF point. $\mathbf{B}_{0}$ has its origin in the period- 4 cardioid $\mathbf{G}_{0}$, $\mathbf{B}_{1}$ in the period- 8 first disc of the period doubling cascade $\mathbf{G}_{1}$, and so on. 